This item was submitted to Loughborough's Research Repository by the author.

Items in Figshare are protected by copyright, with all rights reserved, unless otherwise indicated.

\title{
A novel analytical model of power spectral density function coherent with earthquake response spectra
}

PLEASE CITE THE PUBLISHED VERSION

http://www.eccomas.org/

\section{PUBLISHER}

European Community on Computational Methods in Applied Sciences (ECCOMAS)

\section{VERSION}

AM (Accepted Manuscript)

\section{PUBLISHER STATEMENT}

This work is made available according to the conditions of the Creative Commons Attribution-NonCommercialNoDerivatives 4.0 International (CC BY-NC-ND 4.0) licence. Full details of this licence are available at: https://creativecommons.org/licenses/by-nc-nd/4.0/

\section{LICENCE}

CC BY-NC-ND 4.0

\section{REPOSITORY RECORD}

Barone, Giorgio, Francesco Lo lacono, Giacomo Navarra, and Alessandro Palmeri. 2019. "A Novel Analytical Model of Power Spectral Density Function Coherent with Earthquake Response Spectra". figshare. https://hdl.handle.net/2134/17703. 


\title{
A NOVEL ANALYTICAL MODEL OF POWER SPECTRAL DENSITY FUNCTION COHERENT WITH EARTHQUAKE RESPONSE SPECTRA
}

\author{
Giorgio Barone $^{1}$, Francesco Lo Iacono ${ }^{2}$, Giacomo Navarra $^{2}$ and Alessandro Palmeri ${ }^{1}$ \\ ${ }^{1}$ School of Civil and Building Engineering,Loughborough University \\ Loughborough, Leicestershire, LE11 3TU, United Kingdom \\ e-mail: e-mail: \{G.Barone,A.Palmeri\}@lboro.ac.uk \\ ${ }^{2}$ Facoltà di Ingegneria ed Architettura, Università degli Studi di Enna Kore \\ Cittadella Universitaria - Enna Bassa - 94100 Enna, Italy. \\ e-mail: \{francesco.loiacono,giacomo.navarra\}@ unikore.it
}

Keywords: building code, response spectra, stochastic analysis, Spectrum-compatible PSD.

\begin{abstract}
In the most advanced seismic codes earthquake loads are often defined by means of pseudo-acceleration Response Spectra (RS) and the use of modal superposition analysis method is strongly encouraged. The effectiveness of the design procedures is thus limited by the underlying hypotheses, such as the linearity of the system and the reliability of the modal correlation coefficients used to combine the modal responses for MDOF systems. On the other hand, linear systems response statistics could be easily computed by using stochastic analysis tools, once a stochastic characterization of the seismic action is provided. In this paper a few-parameters analytical model for the definition of Power Spectral Density functions (PSD) coherent with Response Spectra is proposed. Closed-form relationships between the parameters involved in the definition of the PSD and the RS defined by several international seismic codes are provided. The reliability of this tool is assessed by means of a numerical campaign by comparing stochastic analysis and Monte-Carlo simulations. By using the proposed approach, the seismic action can be defined both in terms of RS and in terms of PSD, and, therefore, the engineer can choose the most appropriate analysis tool for his purpose.
\end{abstract}




\section{INTRODUCTION}

The majority of the building codes define the seismic actions by providing the expected maximum response of a single-degree-of freedom (SDOF) system in terms of pseudo-acceleration Response Spectrum (RS). The RS represents the absolute maximum value of a selected response parameter (e.g. displacement, velocity, acceleration) experienced, during the so-called design earthquake, by a SDOF system varying its natural period and for a selected value of its damping ratio. A building code RS curve is determined by the expected SDOF system response at a particular site. It depends on the seismicity of the site, the soil properties, the importance of the structure and, in the most advanced codes, also on the assumed ductility of the lateral load resisting system and the limit state under consideration. The RS analysis is strictly valid only for linear SDOF systems, and its use for the evaluation of the response of MDOF systems implies some approximations [1, 2]. Conversely, a proper probabilistic handling of the assigned seismic action would allow for the full statistical characterization of the response of linear systems in the nodal space, including the response peak distribution [3], avoiding the combination of modal responses and allowing for an effective assessment of the structural reliability. For the case of nonlinear systems, instead, it is always necessary to perform time integration of the structural response with respect to a set of artificially generated ground motion time histories matching the RS (Monte-Carlo simulation). For both cases of linear and nonlinear systems, the development of an analytic tool modelling directly the seismic action is of the utmost importance. It is recognized [4] that the most rigorous way of modelling the seismic excitation is to consider zero-mean Gaussian processes that, under some assumptions, can also be assumed as stationary. The complete probabilistic characterization of the input can be, then, achieved by the knowledge of its Power Spectral Density (PSD) function [5]. It has to be stressed that the PSD is related only to the ground acceleration and it is absolutely independent of the damping ratio of the superimposed structure and by its inherent nonlinearities. The challenge is to set up a robust procedure to define a PSD function compatible with the assigned RS. In the last decades, great attention has been devoted to develop analytic and numeric techniques aiming at obtaining refined models of RS-compatible PSD functions. Earlier contributions on this subject can be found in the review paper by Ahmadi [6]. A common approach to model earthquakes in a stochastic framework is to define filter equations returning the earthquake excitation as response to a white noise. The most used filter is the Tajimi-Kanai one [7, 8]. Falsone et al. [9] provide a technique to obtain the filter coefficients for Eurocode $8 \mathrm{RS}$. Several techniques for generating spectrum-compatible PSDs are available in literature [10, 11]. However, they require iterative procedures and numeric evaluations either in the time domain or in the frequency domain [12, 13]. An analytic model for the evaluation of the PSD compatible with the Eurocode 8 and the former Italian Code RS has been proposed for the first time in [14] and [15], starting from the PSD functions obtained by the numeric procedure proposed in [16]. In this paper, the analytic PSD functions used in [14] and [15] are further generalized to be compatible with a very generic form of RS. In this way, this PSD function can be adopted for a very large range of international seismic codes. The required parameters are analytically evaluated as closed-form functions of the seismic codes RS parameters. The proposed model can be used in place of the RS, so that the practitioner engineer can define the seismic action directly in terms of PSD function and utilize stochastic analysis tools. In the following sections the analytic procedure to evaluate the closed-form expressions relating the RS parameters to the PSD ones is reported. Results are reported for a numerical campaign, performed by means of stochastic analysis on a SDOF system for various building code prescriptions. The results show how the proposed 
analytical form of PSD can be straightforwardly extended to be compatible with various shapes of RS. Additionally, results are confirmed by Monte Carlo simulations.

\section{POWER SPECTRAL DENSITY FUNCTION COMPATIBLE WITH RESPONSE SPEC- TRA}

\subsection{Introduction to the problem of determining the PSD function}

A very general class of pseudo-acceleration RS reported in international building codes can be expressed by means of the following four-branches expression:

$$
S_{a}(T)=\left\{\begin{array}{cc}
S_{0}\left[1+(\alpha-1) \frac{T}{T_{B}}\right] & 0 \leq T \leq T_{B} \\
\alpha S_{0} & T_{B}<T \leq T_{C} \\
\alpha S_{0}\left(\frac{T_{C}}{T}\right)^{k_{1}} & T_{C}<T \leq T_{D} \\
\alpha S_{0}\left(\frac{T_{C}}{T_{D}}\right)^{k_{1}}\left(\frac{T_{D}}{T}\right)^{k_{2}} & T>T_{D}
\end{array}\right.
$$

where $T$ is the natural period of the SDOF system, $S_{0}$ is the peak ground acceleration, $\alpha$ is the dynamic amplification factor, $T_{B}, T_{C}$ and $T_{D}$ are the periods delimitating the various branches, and $k_{1}$ and $k_{2}$ are shape factors. In this paper we refer to RS in which $k_{1}=1$ and $k_{2}=2$. The RS defined by eq. (1) is qualitatively represented in Figure 1. The first branch linearly connects

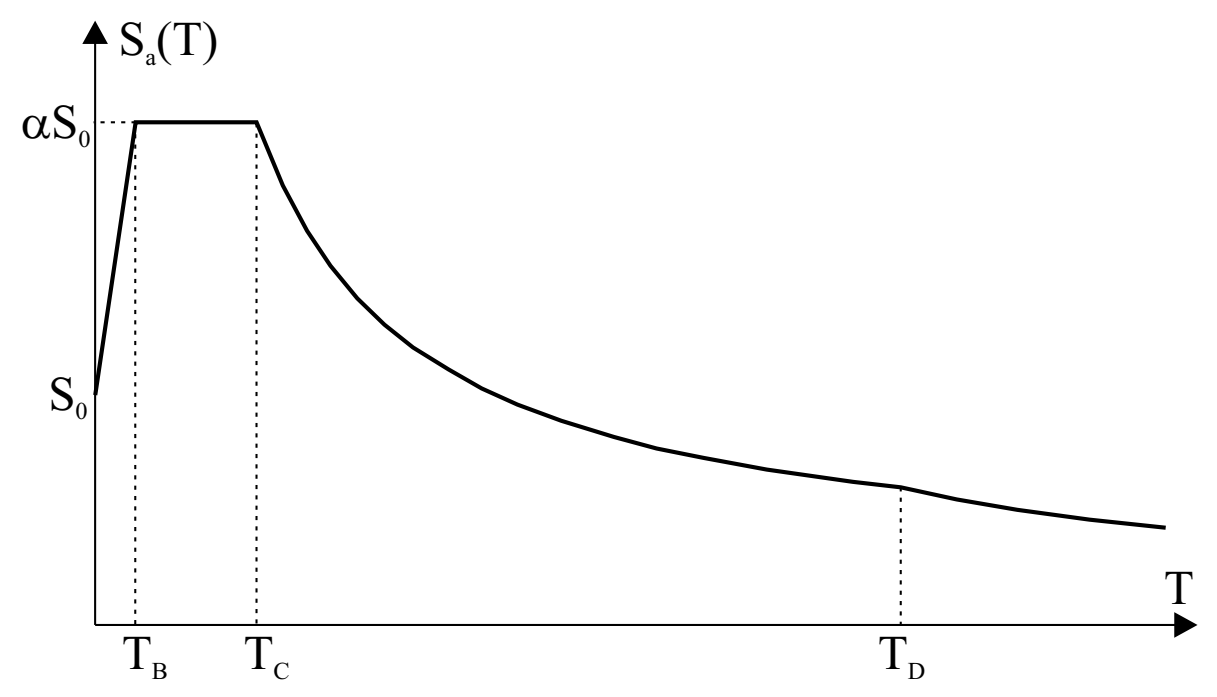

Figure 1: Pseudo-acceleration Response Spectrum.

the point at $T=0$ to the second branch. The second, third and fourth branches of the model corresponds to constant spectral accelerations, velocities and displacements, respectively. Several building codes allow the practitioner engineer to represent the seismic ground motion by means of artificial accelerograms of finite nominal duration $T_{s}$ generated as samples of a zero-mean Gaussian stationary process, fully characterized by its PSD function. However, the seismic codes do not define this PSD function, requiring, instead, that the latter has to be compatible 
with an assigned RS and providing the conditions to achieve the compatibility. In particular, a PSD function $G_{\ddot{U}_{g}}(\omega)$ of the ground acceleration is considered compatible with an assigned acceleration RS, $S_{a}(T)$, if a SDOF system with an assigned damping ratio (usually $\zeta_{0}=0.05$ ), subjected to accelerogram samples generated from $G_{\ddot{U}_{g}}(\omega)$, experiences an absolute maximum acceleration $S_{a}(T)$ for each value of the natural period $T$ into a time window of the nominal duration of the pseudo-stationary part $T_{s}$ of the earthquake. The spectral acceleration can also be expressed as:

$$
S_{a}(\omega, \zeta)=\omega^{2} \eta_{U}(\omega, \zeta) \sigma_{U}(\omega, \zeta)
$$

where $\omega=2 \pi / T$ is the circular frequency, $\sigma_{U}(\omega, \zeta)$ is the standard deviation of the response displacement process and $\eta_{U}(\omega, \zeta)$ is the peak factor, that can be determined as follows [3]:

$$
\eta_{U}(\omega, \zeta)=\sqrt{2 \ln \left\{2 N_{U}(\omega)\left[1-\exp \left(-\delta_{U}^{1.2}(\zeta) \sqrt{\pi \ln \left(2 N_{U}(\omega)\right)}\right)\right]\right\}}
$$

Although the system response is not known a priori, the parameter $N_{U}(\omega)$ and the spread factor $\delta_{U}(\zeta)$ can be expressed by the following approximate equations [17]:

$$
N_{U}(\omega)=-\frac{T_{S}}{2 \pi} \frac{\omega}{\ln (p)} ; \quad \delta_{U}(\zeta)=\sqrt{1-\frac{1}{1-\zeta^{2}}\left[1-\frac{2}{\pi} \arctan \left(\frac{\zeta}{\sqrt{1-\zeta^{2}}}\right)\right]^{2}}
$$

where $p=0.5$, since the mean value of the peak values can be approximated by the $50 \%$ fractile of the maxima distribution. Assuming for the damping ratio $\zeta=0.05$, the spread factor becomes $\delta_{U}\left(\zeta_{0}\right)=0.24561$. Once the PSD of the input is known, the corresponding RS can be easily obtained by eq. (2). However, the inverse problem (i.e. determining the PSD function corresponding to an assigned RS) is not easy to solve due to the strong nonlinearity of eq. (2). To overcome this problem, an approximate expression for the response variance can be used [18] to obtain an estimate of the PSD function $G_{\ddot{U}_{g}}(\omega)$ compatible with the assigned RS:

$$
G_{\ddot{U}_{g}}(\omega)=\frac{\gamma}{\omega}\left[\left(\frac{S_{a}(\omega, \zeta)}{\eta_{U}(\omega, \zeta)}\right)^{2}-\int_{0}^{\omega} G_{\ddot{U}_{g}}(\hat{\omega}) d \hat{\omega}\right]
$$

where the parameter $\gamma=4 \zeta /(\pi-4 \zeta)$ assumes the value $\gamma=0.068$ when $\zeta=0.05$. It is not easy to determine the closed-form solution of eq. (5), since the determination of the PSD function $G_{\ddot{U}_{g}}(\omega)$ at a selected frequency requires the knowledge of the same PSD function for all previous frequencies, that is for each $\hat{\omega} \leq \omega$. A numerical solution of eq. (5) has been provided in [16]. For the numerical implementation of this procedure, the actual ground acceleration PSD is approximated by a constant piecewise function, so that the integral term is replaced by a discrete summation:

$$
G_{\ddot{U}_{g}}\left(\omega_{i}\right)=\frac{4 \zeta}{\pi \omega_{i}-4 \zeta \omega_{i-1}}\left[\left(\frac{S_{a}\left(\omega_{i}, \zeta\right)}{\eta_{U}\left(\omega_{i}, \zeta\right)}\right)^{2}-\Delta \omega \sum_{j=1}^{i-1} G_{\ddot{U}_{g}}\left(\omega_{j}\right)\right]
$$

where $\Delta \omega$ is an arbitrarily selected frequency step, $\omega_{i}=\omega_{0}+(i-0.5) \Delta \omega$, and $\omega_{0}$ is the lower bound of the existence domain of eq. (3). Equation (6) allows to obtain an accurate numerical PSD function compatible with assigned RS. It should be stressed that the effectiveness of this procedure does not depend on the shape of RS and it can be also used for the non-smooth RS obtained in the case of natural earthquake ground motion time histories [19]. 


\subsection{An analytic model for RS compatible PSD function}

Although the method proposed in [16] can be adopted to determine a PSD compatible with any building code RS, the numeric iterative procedure has to be entirely repeated for any variation of the RS parameters. Moreover, the method can be cumbersome for practitioner engineers, who sometimes are not very familiar with the theoretical stochastic aspects on which the definition of the seismic code RS is based. In order to define an analytic PSD function, an extensive numerical campaign has been performed by varying the intensity and shape of the assigned RS, as defined in eq. (1), and evaluating the RS compatible PSD functions using the numeric procedure proposed in [16]. It has been observed that the method always (when $k_{1}=1$ and $k_{2}=2$ ) returns numeric PSD functions having the shape qualitatively reported in Figure 2 . Hence, it

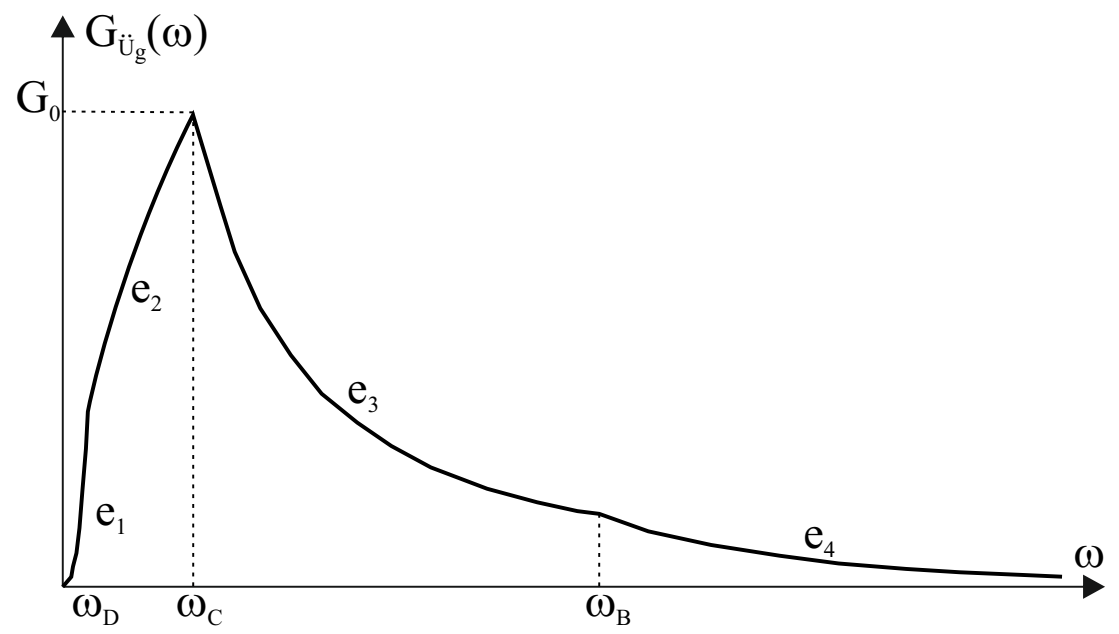

Figure 2: Power Spectral Density function coherent with Response Spectrum.

is straightforward to describe the PSD function as a four-branches piecewise function having a simple mathematical structure and fully defined once few parameters are known. Herein, the following analytical function is proposed:

$$
G_{\ddot{U}_{g}}(\omega)= \begin{cases}G_{0}\left(\frac{\omega_{D}}{\omega_{C}}\right)^{e_{2}}\left(\frac{\omega}{\omega_{D}}\right)^{e_{1}} & 0 \leq \omega \leq \omega_{D} \\ G_{0}\left(\frac{\omega}{\omega_{C}}\right)^{e_{2}} & \omega_{D}<\omega \leq \omega_{C} \\ G_{0}\left(\frac{\omega}{\omega_{C}}\right)^{e_{3}} & \omega_{C}<\omega \leq \omega_{B} \\ G_{0}\left(\frac{\omega_{B}}{\omega_{C}}\right)^{e_{3}}\left(\frac{\omega}{\omega_{B}}\right)^{e_{4}} & \omega>\omega_{B}\end{cases}
$$

where $G_{0}$ represents the peak value of the PSD function at the frequency $\omega=\omega_{C}$. The proposed model is dependent on 5 parameters, namely $G_{0}$ and the four exponents $e_{1}, \ldots, e_{4}$. Therefore, the PSD is fully defined by few parameters. Closed-form expressions for all the parameters can be determined by using eq.(5) in its exact form and taking advantage of some simple considerations, as reported in the following. 


\subsection{Determination of the PSD parameters}

For the sake of clarity, the general form of RS, eq. (1), is herein reported as a function of the circular frequency:

$$
S_{a}(\omega)= \begin{cases}\alpha S_{0}\left(\frac{\omega_{D}}{\omega_{C}}\right)^{k_{1}}\left(\frac{\omega}{\omega_{D}}\right)^{k_{2}} & 0 \leq \omega \leq \omega_{D} \\ \alpha S_{0}\left(\frac{\omega}{\omega_{C}}\right)^{k_{1}} & \omega_{D}<\omega \leq \omega_{C} \\ \alpha S_{0} & \omega_{C}<\omega \leq \omega_{B} \\ S_{0}\left[1+(\alpha-1) \frac{\omega_{B}}{\omega}\right] & \omega>\omega_{B}\end{cases}
$$

To determine the exponent $e_{1}$, the eq. (5) is, at first, rewritten for the frequency $\omega=\omega_{D}$ :

$$
G_{0} \frac{\omega_{D}^{e_{2}+1}}{\omega_{C}^{e_{2}}}=\frac{\gamma}{\omega_{D}}\left[\left(\frac{\alpha S_{0}\left(\frac{\omega_{D}}{\omega_{C}}\right)^{k_{1}}}{\eta_{U}\left(\omega_{D}\right)}\right)^{2}-\int_{0}^{\omega_{D}} G_{\ddot{U}_{g}}(\omega) d \omega\right]
$$

and substituting the eq. (7) into the integral term:

$$
G_{0} \omega_{C}\left(\frac{\omega_{D}}{\omega_{C}}\right)^{e_{2}+1} \frac{\gamma+e_{1}+1}{\gamma\left(e_{1}+1\right)}=\left(\frac{\alpha S_{0}\left(\frac{\omega_{D}}{\omega_{C}}\right)^{k_{1}}}{\eta_{U}\left(\omega_{D}\right)}\right)^{2}
$$

Then, following the same reasoning, but considering a new frequency $\omega=\omega_{D} / \rho(\rho>1)$, the following expression is obtained:

$$
G_{0} \omega_{C}\left(\frac{\omega_{D}}{\omega_{C}}\right)^{e_{2}+1} \frac{\gamma+e_{1}+1}{\gamma\left(e_{1}+1\right)}=\left(\frac{\alpha S_{0}\left(\frac{\omega_{D}}{\omega_{C}}\right)^{k_{1}}}{\eta_{U}\left(\frac{\omega_{D}}{\rho}\right)}\right)^{2} \rho^{e_{1}+1-2 k_{2}}
$$

Comparison of eqs. (10) and (11) leads to:

$$
e_{1}=\frac{\log \frac{\eta_{U}^{2}\left(\frac{\omega_{D}}{\rho}\right)}{\eta_{U}^{2}\left(\omega_{D}\right)}}{\log \rho}+2 k_{2}-1
$$

Moreover, considering the limit $\rho \rightarrow 1$ of eq. (12), it can be demonstrated that the exponent $e_{1}$ can be expressed in closed-form as:

$$
e_{1}=2 k_{2}-1-L\left(\omega_{D}\right)
$$


where the function $L(\omega)$ is defined as:

$$
L(\omega)=2 \omega \frac{d\left(\log \left(\eta_{U}(\omega)\right)\right)}{d \omega}
$$

The evaluation of the closed-form expressions for the other parameters is based on the same concepts, but considering points on the other three branches of the PSD. After some algebra, the following set of parameters is obtained:

$$
\begin{aligned}
& e_{2}=2 k_{1}-1-L\left(\omega_{C}\right) \\
& e_{3}=-1-\gamma-\beta_{2} L\left(\omega_{C}\right) \\
& e_{4}=-1-\gamma-\beta_{3}\left(L\left(\omega_{B}\right)+2 \frac{\alpha-1}{\alpha}\right) \\
& G_{0}=\frac{\gamma}{\beta_{2} \omega_{C}}\left(\frac{\alpha S_{0}}{\eta_{U}^{2}\left(\omega_{C}\right)}\right)^{2}
\end{aligned}
$$

with the following positions:

$$
\begin{aligned}
& \beta_{2}=\left(\frac{\omega_{D}}{\omega_{C}}\right)^{e_{2}+1} \frac{\gamma+e_{1}+1}{e_{1}+1}+\left(1-\left(\frac{\omega_{D}}{\omega_{C}}\right)^{e_{2}+1}\right) \frac{\gamma+e_{2}+1}{e_{2}+1} \\
& \beta_{3}=\left(\frac{\omega_{C}}{\omega_{B}}\right)^{e_{3}+1} \beta_{2}+\left(1-\left(\frac{\omega_{C}}{\omega_{B}}\right)^{e_{3}+1}\right) \frac{\gamma+e_{3}+1}{e_{3}+1}
\end{aligned}
$$

Some seismic codes define the ground motion accelerations by means of three-branches or twobranches RS as follows:

$$
\begin{gathered}
S_{a}(T)= \begin{cases}S_{0}\left[1+(\alpha-1) \frac{T}{T_{B}}\right] & 0 \leq T \leq T_{B} \\
\alpha S_{0} & T_{B}<T \leq T_{C} \\
\alpha S_{0}\left(\frac{T_{C}}{T}\right)^{k_{1}} & T>T_{C}\end{cases} \\
S_{a}(T)=\left\{\begin{array}{cl}
\alpha S_{0} & T \leq T_{C} \\
\alpha S_{0}\left(\frac{T_{C}}{T}\right)^{k_{1}} & T>T_{C}
\end{array}\right.
\end{gathered}
$$

For these case, the PSD model is easily obtained by setting $\omega_{D} \rightarrow 0$ for the first case and $\omega_{D} \rightarrow 0$ and $\omega_{B} \rightarrow \infty$ for the second case, obtaining the following two analytic PSD functions:

$$
G_{\ddot{U}_{g}}(\omega)= \begin{cases}G_{0}\left(\frac{\omega}{\omega_{C}}\right)^{e_{2}} & 0<\omega \leq \omega_{C} \\ G_{0}\left(\frac{\omega}{\omega_{C}}\right)^{e_{3}} & \omega_{C}<\omega \leq \omega_{B} \\ G_{0}\left(\frac{\omega_{B}}{\omega_{C}}\right)^{e_{3}}\left(\frac{\omega}{\omega_{B}}\right)^{e_{4}} & \omega>\omega_{B}\end{cases}
$$




$$
G_{\ddot{U}_{g}}(\omega)=\left\{\begin{array}{cc}
G_{0}\left(\frac{\omega}{\omega_{C}}\right)^{e_{2}} & 0<\omega \leq \omega_{C} \\
G_{0}\left(\frac{\omega}{\omega_{C}}\right)^{e_{3}} & \omega>\omega_{C}
\end{array}\right.
$$

The value of the parameters remain unchanged (taking into account the same positions with regards to $\omega_{D}$ and $\left.\omega_{B}\right)$.

\section{NUMERICAL VALIDATION}

In the present section results have been reported in order to validate the coherency between the PSD function of the seismic action evaluated by means of the proposed analytical model and the assigned RS. Most advanced seismic codes provide the requirements to assess this coherency. In particular, according to Eurocode 8 [20], the coherency is achieved when the 50\% fractile of the distribution of the response peaks of a SDOF system, subjected to the random process characterized by the PSD, is greater than $90 \%$ of the $5 \%$ damping elastic response spectrum, in the range of periods between $0.2 T_{1}$ and $2 T_{1}$, where $T_{1}$ is the fundamental period of the structure. In this paper, this criterion has been adopted, and the response spectrum consistent with the PSD function has been derived through both stochastic analysis and Monte Carlo approach. The response spectrum for a given PSD function of the input can be computed by using eqs. (2) and (3), where the approximation introduced by eq. (4) are removed and the parameters $N_{U}(\omega)$ and $\delta_{U}(\zeta)$ are now replaced by those reported in the following expression:

$$
N_{U}(\omega, \zeta)=\frac{T_{S}}{2 \pi} \sqrt{\frac{\lambda_{U, 2}(\omega, \zeta)}{\lambda_{U, 0}(\omega, \zeta)}}(-\log p)^{-1} ; \quad \delta_{U}(\omega, \zeta)=\sqrt{1-\frac{\lambda_{U, 1}^{2}(\omega, \zeta)}{\lambda_{U, 0}(\omega, \zeta) \lambda_{U, 2}(\omega, \zeta)}}
$$

in which the $\mathrm{i}$-th order spectral moment $\lambda_{U, i}(\omega, \zeta)$ is defined as:

$$
\lambda_{U, i}(\omega, \zeta)=\int_{0}^{\infty} \hat{\omega}^{i}|H(\hat{\omega}, \omega, \zeta)|^{2} G_{\ddot{U}_{g}}(\hat{\omega}) d \hat{\omega}
$$

and the transfer function of the SDOF system is expressed as:

$$
H(\hat{\omega}, \omega, \zeta)=\frac{1}{\omega^{2}-\hat{\omega}^{2}+2 i \zeta \omega \hat{\omega}}
$$

Alternatively, the response spectrum consistent with the PSD function could be evaluated through a Monte Carlo method. Firstly, a certain number of ground acceleration samples are generated as realizations of a zero-mean Gaussian white-noise stationary process having the proposed PSD function. In order to preserve the stationary condition of the response process within a segment of duration $T_{s}$ (i.e. the time-observing window), the time modulation function $\varphi(t)$ proposed in [21] is selected:

$$
\varphi(t)= \begin{cases}\left(t / t_{1}\right)^{2} & t \leq t_{1} \\ 1 & t_{1}<t<t_{2} \\ \exp \left(-\beta\left(t-t_{2}\right)\right) & t \geq t_{2}\end{cases}
$$

with $t_{2}=t_{1}+T_{s}$. Then, the equation of motion of a linear SDOF system characterized by the natural frequency $\omega$ is integrated by means of a step-by-step algorithm and the absolute peak values in terms of displacement, velocity or acceleration can be easily evaluated. Finally, 
the spectral acceleration $S_{a}(\omega, \zeta)$ is computed as the mean value of the acceleration absolute peaks. The acceleration response spectrum curve is obtained by repeating the same procedure for different values of the natural frequency $\omega$, spanning the frequency range of interest. In order to develop some useful applications of the proposed PSD model, a deep research on the different response spectra defined according to several international seismic codes have been performed, choosing countries in such a way to consider both the dimension and the seismicity of the country itself. The RS are then been classified in terms of shape and Table 1 reports the $\mathrm{RS}$ that can be expressed through eq. (1) by letting $k_{1}=1$ and $k_{2}=2$.

\begin{tabular}{|c|c|c|}
\hline Country & seismic code & year \\
\hline \multicolumn{3}{|c|}{ Four-branches RS } \\
\hline Australia & AS1170.4 & 2007 \\
\hline Colombia & NRS10 & 2010 \\
\hline European Union (28 countries) & Eurocode 8 [20] & 2004 \\
\hline \multicolumn{3}{|c|}{ Three-branches RS } \\
\hline Dominican Republic & Decreto 201/11 MOPC & 2011 \\
\hline India & Indian Standard 1893 & 2002 \\
\hline Indonesia & SNI17262002 & 2002 \\
\hline Japan & Building Standard Law & 2000 \\
\hline Korea & Korea Building Code & 2005 \\
\hline Philippines & NSCP & 2010 \\
\hline Taiwan & SDCB & 2005 \\
\hline USA & International Building Code [22] & 2012 \\
\hline \multicolumn{3}{|c|}{ Two-branches RS } \\
\hline Albania & KTP-N.2-89 & 1989 \\
\hline Ecuador & INEN-5 & 2001 \\
\hline Macedonia & Tech. Reg. Code & 1990 \\
\hline Peru & E.030 & 2003 \\
\hline Serbia & Tech. Reg. Code & 1990 \\
\hline Uganda & US 319:2003 & 2003 \\
\hline
\end{tabular}

Table 1: Classification of seismic codes whose RS can be expressed by eq. (1) with $k_{1}=1$ and $k_{2}=2$.

The analytical PSD, in these cases, can be obtained from eq. (7) in which $G_{0}$ and the exponents $e_{i}$ are evaluated as in the previous section. Only major seismic codes are included in reference list for sake of shortness. In the following an application of the proposed model to a four-branches seismic code is proposed. Monte Carlo simulations have been carried out using 2000 samples whose duration is $30 \mathrm{sec}$; the stationary part duration of accelerograms has been assumed $T_{s}=20 \mathrm{sec}$, while, in order to define the modulating function $\varphi(t)$, the value of $t_{1}=5 \mathrm{sec}$ is used. It is worth stressing that the proposed procedure has been successfully tested also for different values of $T_{s}$ and different number of branches. The numerical application is carried out with reference to the four-branches acceleration response spectra defined by the Eurocode 8 [20]. In particular, in order to show the capability of the proposed model to reproduce the assigned RS, the parameters involved in the definition of the RS have been computed according to the Italian Code [23]. According to the Italian Code and Eurocode 8, the acceleration response spectrum has been obtained with reference to a return period of 475 years (10\% probability of exceedance in 50 years) and to a site in Messina (Sicily), soil type A 
(hard rock). The RS parameters have been reported in Table 2, the correspondent PSD function have been evaluated by using eq. (7), and the values of the parameters involved are reported in Table 3. Figure 3 shows the comparison between the PSD function defined by eq. (7) and Table

\begin{tabular}{ll}
\hline Parameter & value \\
\hline$S_{0}$ & $0.248 \mathrm{~g}$ \\
$\alpha$ & 2.411 \\
$T_{B}$ & $0.120 \mathrm{sec}$ \\
$T_{C}$ & $0.359 \mathrm{sec}$ \\
$T_{D}$ & $2.592 \mathrm{sec}$ \\
\hline
\end{tabular}

Table 2: Parameters for the definition of the four-branches response spectrum.

\begin{tabular}{ll}
\hline Parameter & value \\
\hline$G_{0}$ & $1.50932 \cdot 10^{-4} \mathrm{~g}^{2} /(\mathrm{rad} / \mathrm{sec})$ \\
$e_{1}$ & 2.5094 \\
$e_{2}$ & 0.7594 \\
$e_{3}$ & -1.3177 \\
$e_{4}$ & -2.6209 \\
\hline
\end{tabular}

Table 3: Parameters for the definition of the four-branches spectrum-compatible PSD .

3 and the numerical one obtained by eq. (6). The very close agreement between this two curves shows that the analytical model in eq. (7) is able to fit the PSD function computed numerically. The next step is to evaluate the RS related to the PSD reported in Figure 3 and the target RS. This has been investigated both by stochastic analysis and by Monte Carlo simulation and the results have been reported in Figure 4 in terms of acceleration response spectrum, along with the $10 \%$ tolerance curve. Figure 4 shows that the proposed analytical PSD is able to reproduce the given response spectrum with an error much lower than the allowed tolerance in case of both stochastic analysis and Monte Carlo simulation. Similar result can be obtained for the other RS listed in Table 1.

\section{CONCLUSIONS}

Most of the response spectra in the building codes of the various countries are characterized by having branches in the period domain represented by linear, constant and power laws in the different period lag. The number of branches, the intensities and the various exponents depend on the country, the seismicity of the various zones as well as on the particular site conditions. In this paper, the authors have proposed a single analytical expression of the PSD coherent with the response spectra in all different scenarios. The model can be used for a segmented input-output stationary process for different conventional durations of the earthquake. Maximum peak analysis is performed via Monte Carlo simulations of artificial accelerograms generated by the PSD coherent with the response spectrum at hand. Alternatively, for linear structural systems, the maxima may be evaluated by taking full advantage of stochastic analysis. Coherency between the proposed PSDs and response spectra with four, three and two branches has been validated by extensive use of Monte Carlo simulation method obtaining errors much lower than the $10 \%$ prescribed tolerance. 


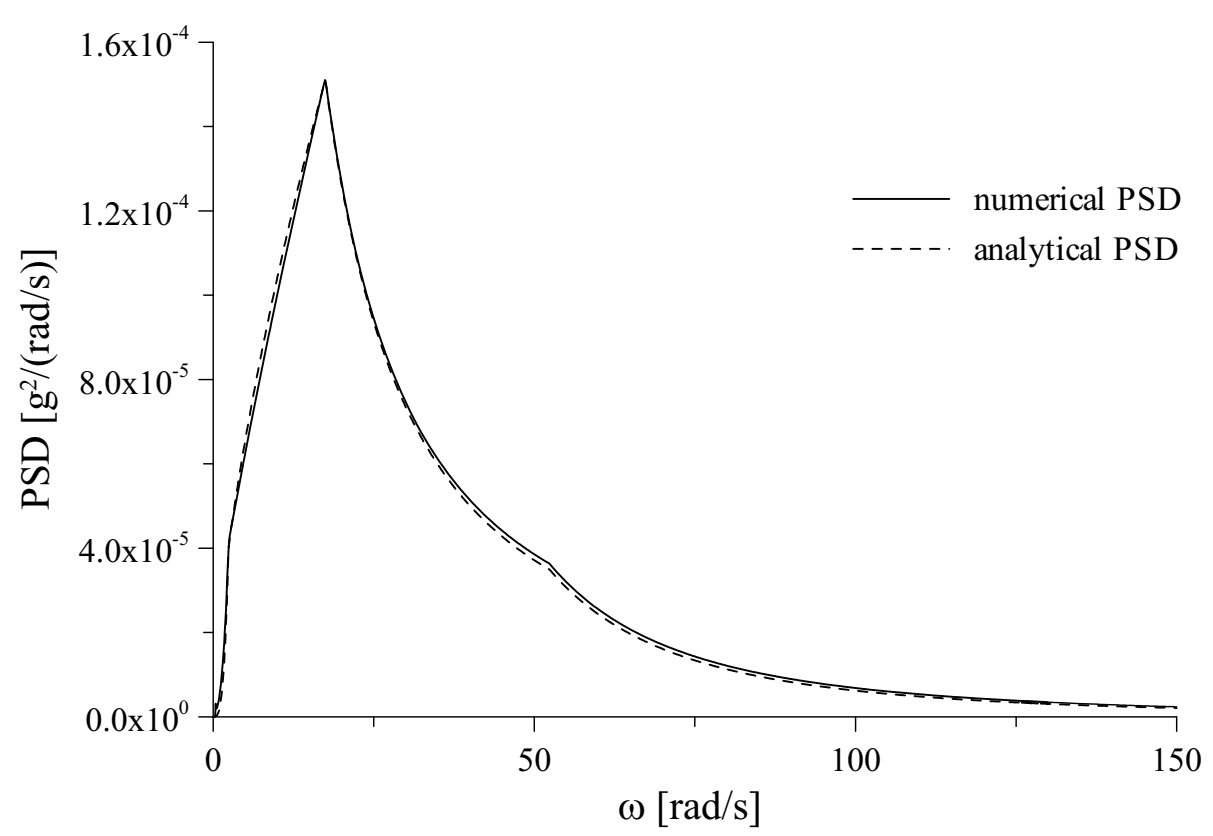

Figure 3: PSD compatible with four-branches RS; numerical PSD obtained by eq. 67 (solid line); PSD defined by eq. (7) and Table 3 (dashed line).

\section{REFERENCES}

[1] E. Rosenblueth, A basis for aseismic design, PhD thesis, University. of Illinois, Urbana, IL,USA, 1951.

[2] E.L. Wilson, A. Der Kiureghian, E.P. Bayo, A replacement for the SRSS method in seismic analysis. Earthquake Engineering and Structural Dynamic, 9, 187-192, DOI:10.1002/eqe.4290090207, 1981.

[3] E.H. Vanmarcke, Properties of spectral moments with applications to random vibration. Journal of Engineering Mechanics, 98(2), 425-446, 1972.

[4] R.W. Clough, J. Penzien, Dynamics of structures, 2nd edition, McGraw-Hill, New York, 1993.

[5] Y.K. Lin, Probabilistic Theory of Structural Dynamics, McGraw-Hill, New York,1967.

[6] G. Ahmadi, Generation of artificial time-histories compatible with given response spectra a review, SM Archives, 4(3), 207-239, 1979.

[7] K. Kanai, Semi-empirical formula for the seismic characteristics of the ground, Univ. Tokyo Bull. Earthquake Res. Inst., 35, 309-325, 1957.

[8] H. Tajimi, A statistical method of determining the maximum response of a building structure during an earthquake, Proc. $2 d$ World Conf. Earthquake Eng., Tokyo and Kyoto, 11, 781-798, 1960.

[9] G. Falsone, G. Neri, Stochastic modelling of earthquake excitation following the EC8: power spectrum and filtering equations. European Earthquake Engineering, 1, 3-12, 2000. 


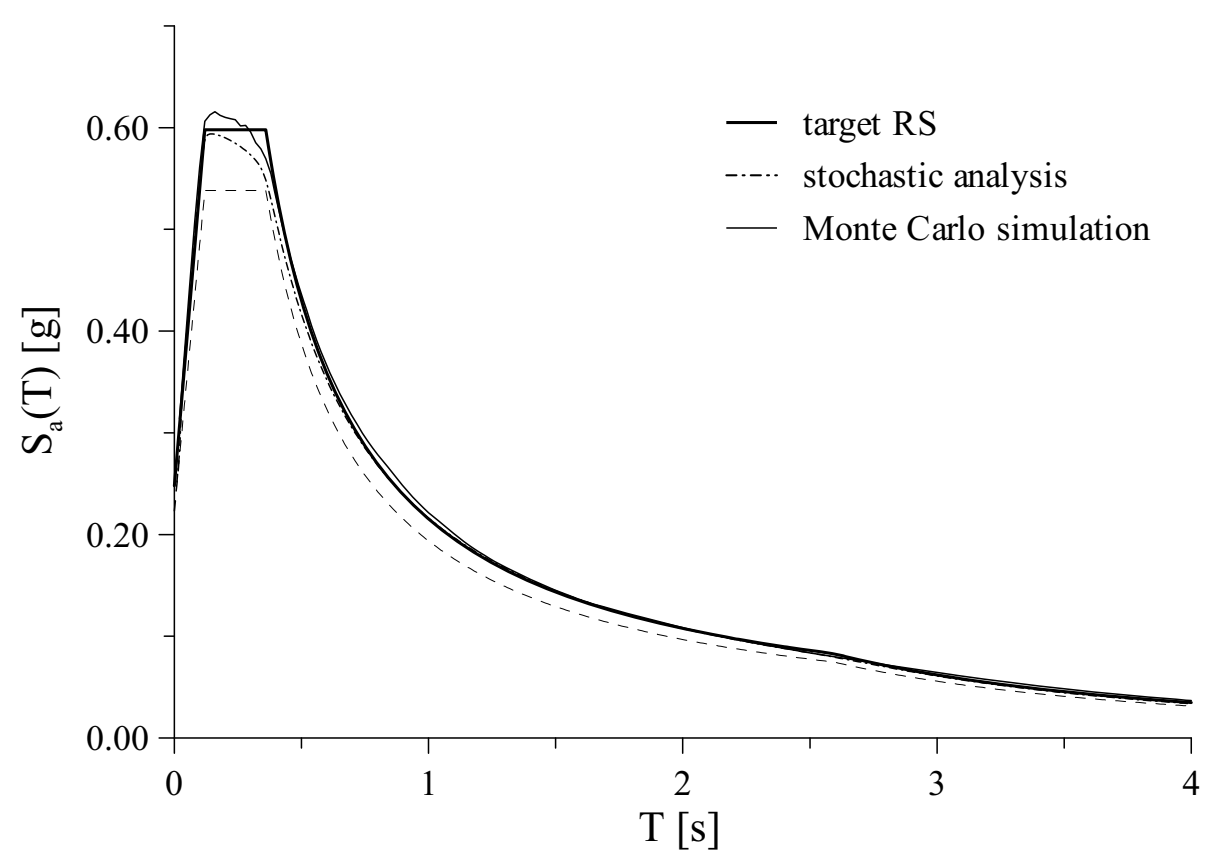

Figure 4: Four-branches acceleration RS; target RS defined by eq. (1) and Table 3 (bold line) and 10\% tolerance curve (dashed line); validation RS obtained by stochastic analysis (dash-dot line); validation RS obtained by Monte Carlo simulation (solid line).

[10] D.D. Pfaffinger, Calculation of power spectra from response spectra, Journal of Engineering Mechanics, 109, 357-372, 1983.

[11] A. Preumont, The generation of spectrum compatible accelerograms for the design of nuclear power plants. Earthquake Engineering and Structural Dynamics, 12, 481-497, 1983.

[12] M. Kaul, Stochastic characterization of earthquakes through their Response Spectrum. Earthquake Engineering and Structural Dynamics, 6, 497-509, 1978.

[13] J.F. Urnuh, D.D. Kana, An iterative procedure for the generation of consistent power/response spectrum. Nuclear Engineering and Design, 66, 427-435, 1981.

[14] M. Di Paola, L. La Mendola, G. Navarra, Stochastic seismic analysis of structures with nonlinear viscous dampers. Journal of Structural Engineering - ASCE, 133(10), 14751478, 2007.

[15] M. Di Paola, G. Navarra, Stochastic seismic analysis of MDOF structures with nonlinear viscous dampers. Structural Control and Health Monitoring, 16, 303-318, 2009.

[16] P. Cacciola, P. Colaianni, G. Muscolino, Combination of Modal Responses Consistent with Seismic Input Representation. Journal of Structural Engineering ASCE, 130(1), 4755, 2004.

[17] A. Der Kiureghian, Structural response to stationary oscillation. Journal of Engineering Mechanics, 106(6), 1195-1213, 1980.

[18] E.H. Vanmarcke, Structural response to earthquakes, C. Lominitz and E. Rosenblueth, eds., Seismic risk and engineering decisions, 287-337, Elsevier, New York, 1977. 
[19] P. Cacciola, A stochastic approach for generating spectrum compatible fully nonstationary eathquakes. Computers \& Structures, 88, 889-901, 2010.

[20] UNI ENV 1998:2005, Eurocode 8: Design of structures for earthquake resistance Part 1: General rules, seismic actions and rules for buildings, CEN Central Secretariat, Brussels, Belgium, 2005.

[21] P.C. Jennings, G.W. Housner, C. Tsai, Simulated earthquake motions for design purpose. Proceedings of the 4th World Conference on Earthquake Engineering, A-1, 145-160, Santiago, Chile, 1969.

[22] International Code Council, 2012 International Building Code, Country Club Hills, Ill: ICC, 2011.

[23] Ministero delle Infrastrutture e dei trasporti, D.M. 14.01.2008 - Norme tecniche per le Costruzioni, in Italian, Roma, Italy, 2008 